\title{
Extremely high prevalence of multi-resistance among uropathogens from hospitalised children in Beira, Mozambique
}

B T van der Meeren, ${ }^{1}$ MSc; K D Chhaganlal, ${ }^{2}$ MD; A Pfeiffer, ${ }^{3}$ MD; E Gomez, ${ }^{2}$ MD; J J Ferro, ${ }^{3}$ MD; M Hilbink, ${ }^{4}$ PhD; C Macome, ${ }^{2}$ MD; F J van der Vondervoort, ${ }^{5} \mathrm{BSc}$; K Steidel, ${ }^{3} \mathrm{MD}$; P C Wever, ${ }^{5} \mathrm{MD}, \mathrm{PhD}$

\author{
Universidade Católica de Moçambique, Beira, Mozambique, and Department of Medical Microbiology and Infection Control, Jeroen Bosch \\ Hospital, 's-Hertogenbosch, The Netherlands \\ ${ }^{2}$ Hospital Central da Beira, Beira, Mozambique \\ ${ }^{3}$ Universidade Católica de Moçambique, Beira, Mozambique \\ ${ }^{4}$ Jeroen Bosch Academy, Jeroen Bosch Hospital, 's-Hertogenbosch, The Netherlands \\ ${ }^{5}$ Department of Medical Microbiology and Infection Control, Jeroen Bosch Hospital, 's-Hertogenbosch, The Netherlands
}

Corresponding author: B van der Meeren (gittavdmeeren@hotmail.com)

\begin{abstract}
Objectives. A prospective surveillance study was conducted to investigate the epidemiology and patterns of antibiotic resistance among uropathogens from hospitalised children in Beira, Mozambique. Additionally, information regarding determinants of a urinary tract infection (UTI) was obtained.

Methods. Bacterial species identification, antimicrobial susceptibility testing and extended-spectrum beta-lactamase testing were performed for relevant bacterial isolates.

Results. Analysis of 170 urine samples from 148 children yielded 34 bacterial isolates, predominantly Escherichia coli and Klebsiella spp., causative of a urinary tract infection in 29 children; 30/34 isolates (88.2\%) from 26/29 children (89.7\%) were considered highly resistant micro-organisms (HRMOs). No significant determinants of urinary tract infection with HRMOs were detected when analysing gender, antibiotic use during hospital admission and HIV status.

Conclusion. This study shows, for the first time in Mozambique, an extremely high prevalence of HRMOs among uropathogens from hospitalised children with a urinary tract infection.
\end{abstract}

S Afr Med J 2013;103(6):382-386. DOI:10.7196/SAMJ.5941

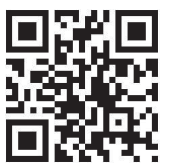

Bacteria are champions of evolution, and microbes have adapted to a point where they pose serious clinical challenges for humans. ${ }^{[1]}$ Emergence of antimicrobial resistance among bacteria is a worldwide problem. New mechanisms of bacterial resistance that have been detected include penicillin-binding protein defects, extendedspectrum beta-lactamases (ESBLs) and metallo-beta-lactamases. ${ }^{[2,3]}$ Guidelines for first-line antibiotics in the treatment of different infectious diseases have an empiric basis, but recommended antibiotics are not always as successful as they are expected to be. Collection of microbial data on a case-by-case basis is often necessary in choosing effective individual antimicrobial therapy, but scarcity of microbiology laboratory capacity in developing countries limits this approach. In these countries, the best option would be to base antimicrobial therapy on local data, if available, regarding the susceptibility of common pathogens to antibiotics. ${ }^{[4]}$

Mozambique, one of the poorest countries of the world, has a shortage of microbiology laboratory facilities, and choice of antimicrobial therapy often is guided solely by availability of antibiotics. Few studies have addressed the emergence of antimicrobial-resistant bacteria in Mozambique. Increasing resistance to chloramphenicol among blood culture isolates of non-typhi Salmonella species, Escherichia coli, Staphylococcus aureus and Haemophilus influenzae has been reported. ${ }^{\left[{ }^{[5]}\right.}$ Likewise, high resistance to tetracycline was reported among Neisseria gonorrhoeae isolates, while diarrhoeagenic E. coli isolates showed high resistance to trimethoprim-sulphamethoxazole and ampicillin. ${ }^{[6,7]}$
Urinary tract infection (UTI) is one of the most common bacterial infections encountered by clinicians in developing countries and the cause of significant morbidity and mortality. ${ }^{[8]}$ Several studies from the African continent have investigated the profile of common uropathogens and the pattern of their susceptibility to commonly used antimicrobial agents in order to guide choice of empiric therapy. These studies reported the emergence of antibiotic-resistant Gram-negative bacilli with special emphasis on ESBL-producing isolates. ${ }^{[9-11]}$

To our knowledge, no studies have been published describing antimicrobial resistance among uropathogens in Mozambique. A small pilot study of the antimicrobial susceptibility pattern of common uropathogens conducted at the Universidade Católica de Moçambique (UCM) showed results warranting further investigation. A prospective surveillance study was therefore conducted with the aim of investigating the epidemiology and patterns of antibiotic resistance of uropathogens from hospitalised children at the Hospital Central da Beira (HCB).

\section{Materials and methods Study population}

The study was conducted from 8 October to 10 December 2009 in the two paediatric wards of $\mathrm{HCB}$, a 728-bed referral hospital in Beira, the second-largest city in Mozambique. A maximum of 150 and 60 children are treated in the 100-bed paediatric ward and the 24-bed malnutrition ward, respectively; one bed is therefore often occupied by more than one child. One family member is allowed to stay in the 
hospital to take care of the child. For each child included in the study, information regarding age, gender, antibiotic use during hospital admission and HIV status was recorded on a data information sheet. The study was approved by the local medical ethical committee.

\section{Microbiological data}

Laboratory investigations were all performed locally in UCM facilities in Beira. Nurses were instructed to provide midstream clean-catch urine specimens, and to use urine collection bags in the case of children who were not toilet trained. Suprapubic bladder aspiration was considered too invasive. All urine samples from the paediatric and malnutrition wards were collected in sterile containers and analysed within the hour using a commercial urine dipstick test (Combur 8 Test, Boehringer Mannheim GmbH, Mannheim, Germany). In the paediatric ward, urine analysis was requested if UTI was suspected on clinical grounds, whereas it was routinely performed in the malnutrition ward, typically within a few days after admission. Urine samples for which leucocyte esterase and/or nitrite tests were positive were inoculated on blood agar plates (Becton, Dickinson \& Co., Sparks, MD, USA) and eosin methylene blue agar plates (Becton, Dickinson \& Co.), followed by incubation for $18-24$ hours at $35^{\circ} \mathrm{C}$. The same process was undertaken when leucocyte esterase and nitrite tests were negative on dipstick analysis of turbid urine samples but Gram staining, only performed in these cases, showed leucocytes and/or bacteria. Bacterial growth was evaluated according to American Society for Microbiology guidelines. ${ }^{[12]}$ Relevant bacterial isolates were identified using commercial identification strips (API 20E and API 20NE systems, bioMérieux, Marcy l'Etoile, France). Antimicrobial susceptibility testing (AST) was performed by disc diffusion on Mueller-Hinton II agar plates (Becton, Dickinson \& Co.) with an inoculum of 0.5 McFarland, using Neo-Sensitabs tablets (Rosco Diagnostica, Taastrup, Denmark). Inhibition zones were interpreted according to Clinical and Laboratory Standard Institute guidelines. For Gram-negative isolates, the following Neo-Sensitabs tablets were examined: amoxicillin $30 \mu \mathrm{g}$, piperacillin $100 \mu \mathrm{g}$, amoxicillin/ clavulanate $30 / 15 \mu \mathrm{g}$, piperacillin/tazobactam $100 / 10 \mu \mathrm{g}$, cefazolin $60 \mu \mathrm{g}$, cefuroxime $60 \mu \mathrm{g}$, cefotaxime $30 \mu \mathrm{g}$, cefotaxime/clavulanate $30 / 10 \mu \mathrm{g}$, ceftazidime $30 \mu \mathrm{g}$, ceftazidime/clavulanate $30 / 10 \mu \mathrm{g}$, cefepime $30 \mu \mathrm{g}$, cefepime/clavulanate $30 / 10 \mu \mathrm{g}$, imipenem $15 \mu \mathrm{g}$, amikacin $40 \mu \mathrm{g}$, kanamycin $100 \mu \mathrm{g}$, gentamicin $40 \mu \mathrm{g}$, nalidixic acid $130 \mu \mathrm{g}$, ciprofloxacin $10 \mu \mathrm{g}$, nitrofurantoin $260 \mu \mathrm{g}$, fosfomycin $70 \mu \mathrm{g}$, trimethoprim/sulfamethoxazole $5.2 / 240 \mu \mathrm{g}$, chloramphenicol $60 \mu \mathrm{g}$, doxycycline $80 \mu \mathrm{g}$ and tetracycline $80 \mu \mathrm{g}$. The following Neo-Sensitabs tablets were examined for Gram-positive isolates: amoxicillin $30 \mu \mathrm{g}$, amoxicillin/clavulanate $30 / 15 \mu \mathrm{g}$, cefoxitin $60 \mu \mathrm{g}$, ciprofloxacin $10 \mu \mathrm{g}$, nitrofurantoin $260 \mu \mathrm{g}$, chloramphenicol $60 \mu \mathrm{g}$, doxycycline $80 \mu \mathrm{g}$, clindamycin $25 \mu \mathrm{g}$, erythromycin $78 \mu \mathrm{g}$ and vancomycin $5 \mu \mathrm{g}$. According to current Dutch guidelines, strains producing ESBL were defined as strains showing an increase of $5 \mathrm{~mm}$ or more in inhibition zones with cefotaxime/clavulanate and/ or ceftazidime/clavulanate and/or cefepime/clavulanate, compared with zones with their clavulanate-free counterparts. An ESBL-positive isolate was considered resistant to all penicillins and cephalosporins tested. ${ }^{[13]}$ The guideline Measures to Prevent Transmission of Highly Resistant Microorganisms (HRMOs), published in 2005 by the Dutch Working Party on Infection Prevention, was used to identify HRMOs in cultured isolates. ${ }^{[14]}$

\section{Statistical analysis}

Logistic regression was performed to investigate the relationships between gender, antibiotic use during hospital admission and HIV status (determinants) on the one hand and UTI with HRMOs (outcome variable) on the other hand. Two analyses were performed for these relationships: a crude analysis and an adjusted analysis. A $p$-value of $<0.05$ (two-tailed) was considered to indicate statistical significance. Statistical analyses were performed using PASW 18.0 for Windows (SPSS Inc., Chicago, IL, USA).

\section{Results}

\section{Malnutrition ward}

From the malnutrition ward, 88 urine samples were received from 76 children ( 47 males and 29 females; $61.8 \%$ and $38.2 \%$ ). The median age was 2 years (range 3 months - 13 years). HIV status was documented in 62 of 76 children, of whom 29 were HIV-positive (46.8\%; 18 males and 11 females). Table 1 shows the antibiotics used during hospital stay of 75 of the 76 children admitted to the malnutrition ward.

Dipstick analysis and Gram staining, the latter performed on 11 samples, identified 56 urine samples for subsequent culture from 46 children ( 26 males and 20 females). Culture yielded 19 bacterial isolates causative of UTI from 16 urine samples from 16 children (21.1\% of all studied children; 10 males and 6 females). Cultured micro-organisms included E. coli $(n=11)$ and Klebsiella spp. $(n=8)$. Table 2 shows the AST results for these two pathogens.

\section{Paediatric ward}

From the paediatric ward, 82 urine samples were received from 72 children (41 males and 30 females; $57.7 \%$ and $42.3 \%$; gender unknown for 1 child). The median age was 5 years (range 6 months 12 years). HIV status was documented in 70 of 72 children, of whom 14 were HIV-positive (20.0\%; 8 males and 6 females). Table 1 shows the use of antibiotics during hospital stay of 72 children admitted to the paediatric ward.

Dipstick analysis and Gram staining, the latter performed on 6 samples, identified 46 urine samples for subsequent culture from 44 children (19 males and 24 females; gender unknown for 1 child). Culture yielded 15 bacterial isolates causative of a UTI from 13 urine samples from 13 children (18.1\% of studied individuals; 8 males and 4 females; gender unknown for 1 child). Cultured micro-organisms included E. coli ( $n=3)$, Klebsiella spp. ( $n=9)$, Proteus mirabilis $(n=1)$, Citrobacter freundii $(n=1)$ and Enterococcus spp. $(n=1)$. Table 2 shows the AST results for the two predominant pathogens, E. coli and Klebsiella spp.

Table 1. Use of antibiotics during hospital stay of children admitted to the malnutrition and paediatric wards

\begin{tabular}{lll}
\hline & $\begin{array}{l}\text { Malnutrition ward } \\
(\boldsymbol{N}=75)^{*} \\
\boldsymbol{n}(\%)\end{array}$ & $\begin{array}{l}\text { Paediatric ward } \\
(\boldsymbol{N}=\mathbf{7 2}) \\
\boldsymbol{n}(\%)\end{array}$ \\
\hline Penicillin G & $20(26.7)$ & $33(45.8)$ \\
Amoxicillin & $44(58.7)$ & $16(22.2)$ \\
Ceftriaxone & $5(6.7)$ & $3(4.2)$ \\
Gentamicin & $25(33.3)$ & $5(6.9)$ \\
Ciprofloxacin & $3(4.0)$ & $0(0.0)$ \\
Trimethoprim/ & $31(41.3)$ & $3(4.2)$ \\
sulfamethoxazole & & $17(23.6)$ \\
Chloramphenicol & $34(45.3)$ & $3(4.2)$ \\
Erythromycin & $0(0.0)$ & $0(0.0$ \\
Metronidazole & $6(8.0)$ &
\end{tabular}

${ }^{*}$ Antibiotic use was not documented in 1 of 76 children admitted to the malnutrition ward. 
Table 2. Resistance to antibiotics ${ }^{\star}$ among Escherichia coli and Klebsiella spp. isolates from urine samples of children admitted to the malnutrition and paediatric wards

\begin{tabular}{|c|c|c|c|c|}
\hline & \multicolumn{2}{|c|}{ Malnutrition ward } & \multicolumn{2}{|c|}{ Paediatric ward } \\
\hline & $\begin{array}{l}\text { E. coli }(N=11) \\
n(\%)\end{array}$ & $\begin{array}{l}\text { Klebsiella spp. }(N=8) \\
n(\%)\end{array}$ & $\begin{array}{l}\text { E. } \operatorname{coli}(N=3) \\
n(\%)\end{array}$ & $\begin{array}{l}\text { Klebsiella spp. (N=9) } \\
n(\%)\end{array}$ \\
\hline Amoxicillin & $11(100.0)$ & $8(100.0)$ & $3(100.0)$ & $9(100.0)$ \\
\hline Piperacillin & $11(100.0)$ & $8(100.0)$ & $3(100.0)$ & $9(100.0)$ \\
\hline Amoxicillin/clavulanate & $7(63.6)$ & $6(75.0)$ & $2(66.7)$ & $9(100.0)$ \\
\hline Piperacillin/tazobactam & $10(90.9)$ & $7(87.5)$ & $2(66.7)$ & $9(100.0)$ \\
\hline Cefazolin & $9(81.8)$ & $6(75.0)$ & $3(100.0)$ & $9(100.0)$ \\
\hline Cefuroxime & $7(63.6)$ & $6(75.0)$ & $2(66.7)$ & $9(100.0)$ \\
\hline Cefotaxime & $7(63.6)$ & $6(75.0)$ & $2(66.7)$ & $9(100.0)$ \\
\hline Ceftazidime & $7(63.6)$ & $6(75.0)$ & $2(66.7)$ & $9(100.0)$ \\
\hline Cefipime & $7(63.6)$ & $6(75.0)$ & $2(66.7)$ & $9(100.0)$ \\
\hline Imipenem & $0(0.0)$ & $0(0.0)$ & $0(0.0)$ & $0(0.0)$ \\
\hline Amikacin & $0(0.0)$ & $0(0.0)$ & $0(0.0)$ & $0(0.0)$ \\
\hline Kanamycin & $5(45.5)$ & $4(50.0)$ & $1(33.3)$ & $5(55.6)$ \\
\hline Gentamicin & $9(81.8)$ & $7(87.5)$ & $2(66.7)$ & $8(88.9)$ \\
\hline Nalidixic acid & $9(81.8)$ & $3(37.5)$ & $2(66.7)$ & $3(33.3)$ \\
\hline Ciprofloxacin & $7(63.6)$ & $3(37.5)$ & $1(33.3)$ & $2(22.2)$ \\
\hline Nitrofurantoin & $1(9.1)$ & $2(25.0)$ & $0(0.0)$ & $1(11.1)$ \\
\hline Fosfomycin & $0(0.0)$ & $0(0.0)$ & $0(0.0)$ & $1(11.1)$ \\
\hline Trimethoprim/sulfamethoxazole & $11(100.0)$ & $8(100.0)$ & $3(100.0)$ & $8(88.9)$ \\
\hline Chloramphenicol & $10(90.9)$ & $7(87.5)$ & $3(100.0)$ & $9(100.0)$ \\
\hline Doxycycline & $8(72.7)$ & $3(37.5)$ & $2(66.7)$ & $5(55.6)$ \\
\hline Tetracycline & $9(81.8)$ & $3(37.5)$ & $3(100.0)$ & $5(55.6)$ \\
\hline ESBL production & $7(63.6)$ & $6(75.0)$ & $2(66.7)$ & $9(100.0)$ \\
\hline Combined aminoglycoside/quinolone resistance & $9(81.8)$ & $3(37.5)$ & $2(66.7)$ & $3(33.3)$ \\
\hline HRMO & $9(81.8)$ & $7(87.5)$ & $3(100.0)$ & $9(100.0)$ \\
\hline
\end{tabular}

\section{Highly resistant micro-organisms}

Overall, analysis of 170 urine samples from 148 children yielded 34 bacterial isolates, predominantly E. coli and Klebsiella spp., causative of a UTI in 29 children. According to Dutch guidelines, $30 / 34$ isolates (88.2\%) from 26/29 children (89.7\%) were considered HRMOs on the basis of ESBL production $(n=26)$, combined resistance to aminoglycosides and quinolones in E. coli and Klebsiella spp. ( $n=17)$, and combined resistance to aminoglycosides, quinolones and trimethoprim/sulfamethoxazole in P. mirabilis and C. freundii $(n=2)$.
Determinants of urinary tract infection with highly resistant micro-organisms

In the crude analysis, gender, which was known for 147 of 148 children, was not associated with UTI with HRMOs (odds ratio (OR) 0.66 ; $95 \%$ confidence interval (CI) $0.26-1.63 ; p=0.37$ ). Information on antibiotic use during hospital admission was obtained for 147 of 148 children. In children receiving either $0-2(n=119)$ or $3-4$ $(n=28)$ different antibiotics during admission, UTI with HRMOs was found in $19(16.0 \%)$ and 7 (25.0\%) children, respectively. Children receiving 3 - 4 different antibiotics during admission did 
not have a significantly higher risk of UTI with HRMOs (OR 1.75; $95 \%$ CI $0.65-4.70 ; p=0.26$ ) compared with children receiving 0 - 2 antibiotics. HIV status was documented for 132 of 148 children. In HIV-negative children $(n=89)$ and HIV-positive children ( $n=43)$, UTI with HRMOs was found in 14 (15.7\%) and 7 (16.3\%) children, respectively. HIV-positive children did not have a higher risk of UTI with HRMOs (OR 1.04; 95\% CI $0.39-2.81 ; p=0.94$ ) compared with HIV-negative children.

\section{Discussion}

This study shows, for the first time in Mozambique, an extremely high prevalence of HRMOs in Gram-negative uropathogens from hospitalised children with a UTI. The recommended antibiotic for treatment of UTI in children hospitalised at HCB is currently amoxicillin, which as this study shows cannot be expected to be clinically successful and should be reconsidered. Likewise, in neighbouring South Africa, it has been recommended that because of high resistance levels among Gram-negative uropathogens, amoxicillin and trimethoprim/sulfamethoxazole should not be used for empiric treatment of UTI at Dr George Mukhari Hospital in Ga-Rankuwa, Pretoria. ${ }^{[11]}$

A prevalence of $15-20 \%$ resistance to an antibiotic has been considered an acceptable threshold for the antibiotic to be included in empiric coverage. ${ }^{[15]}$ Suitable empiric options for treatment of UTI in hospitalised children at HCB therefore include amikacin, imipenem, nitrofurantoin and fosfomycin. However, these antibiotics are either not available in Mozambique, inappropriate because of broad-spectrum antimicrobial activity, restricted for use in uncomplicated UTI, or require blood level monitoring because of side-effects. Both empiric and directed antimicrobial treatment of these UTIs is therefore a major clinical challenge in Mozambique. It is, however, important to note that the study subjects were hospitalised children, most of whom were receiving treatment with multiple antibiotics. Presumably our results are not representative of HRMOs in outpatients or the general population of the country, and it would be of value to investigate the prevalence in both these groups. Such studies are, however, hampered by our lack of adequate clinical microbiology facilities. The absence of such facilities also means that we are not informed about the frequency of recurrent UTI in the study population. Likewise, renal imaging by ultrasound to look for urinary tract anomalies is seldom performed. However, in a study of malnourished black children in South Africa no anatomical abnormalities were demonstrated in a population with a high prevalence of UTI. ${ }^{[16]}$

In this study, no resistance was found to amikacin despite the high level of resistance to the other aminoglycosides tested, kanamycin and gentamicin. This divergence is consistent with previously reported results from the African continent. ${ }^{[17]}$ Amikacin is a derivative of kanamycin A with the amino group at position 1 acylated by 4 -amino-2-hydroxybutyrate. The high activity of amikacin in comparison with other aminoglycosides is attributed to the presence of the aminohydroxybutyryl group, which generally prevents the enzymatic modification of amikacin. ${ }^{[18]}$

It has been stated that heavy use and misuse of antibiotics are major causes of the high numbers of resistant pathogenic and commensal bacteria observed worldwide. ${ }^{[19]}$ We found that the risk of UTI with HRMOs has an OR of 1.75 in children receiving 3 - 4 different antibiotics during admission, compared with children receiving 0 - 2 antibiotics. This did not reach significance, however, presumably owing to low power as a result of small patient numbers. High antibiotic use was observed, especially in the malnutrition ward. This is in accordance with World Health Organization recommendations ${ }^{[20]}$ in which oral treatment with trimethoprim/sulfamethoxazole is suggested for malnourished children with no apparent signs of infection and no complications, while systemic treatment with amoxicillin and gentamicin is suggested for malnourished children with complications such as septic shock, hypoglycaemia, hypothermia, or skin, respiratory or urinary tract infections, or for those who appear lethargic or sickly. In view of the worldwide development of antimicrobial resistance and its relation to heavy use and misuse of antibiotics, the clinical practice of prescribing antibiotics to malnourished children without an apparent infection seems debatable, but is explained by the fact that malnourished children with serious infections may not display fever and inflammation. ${ }^{[20]}$

There is little in the literature on the impact of HIV on the presentation of UTI in children. We were unable to show an association between HIV status and the presence of UTI with HRMOs in hospitalised children, in keeping with previous results showing no significant effect of HIV on the presentation of UTI in children. ${ }^{[21]}$ We did not investigate the relationship between age and UTI with HRMOs because of the great variation in age in children admitted to the malnutrition and paediatric wards.

In this study, we observed little use of third-generation cephalosporins such as ceftriaxone in either ward. Nevertheless, we observed high-level resistance to third-generation cephalosporins due to ESBL production. Currently there is great concern about the possible relationship between heavy antibiotic use in chicken farming and the presence of ESBL-producing Gram-negative bacilli in humans. ${ }^{[2]}$ Studies of antimicrobial resistance of Gram-negative bacilli in poultry in Mozambique could give insight into the origin of the observed ESBL-producing strains in humans. Nosocomial cross-contamination has also been reported as a major mode of acquisition of multi-resistant bacteria, especially among hospitalised patients being treated with antibiotics. ${ }^{[23]}$ Polymerase chain reaction techniques would be required to characterise resistance genes and determine clonal relatedness of the isolated HRMOs in our study. However, whether or not clonal relatedness is apparent, the role of infection control practices in this situation cannot be overemphasised.

In conclusion, our results show an extremely high prevalence of HRMOs among uropathogens from hospitalised children with a UTI in Beira, Mozambique.

Acknowledgements. We thank the nurses of the malnutrition and paediatric wards and the laboratory technicians of the Hospital Central da Beira for their full co-operation.

Funding sources. None.

\section{References}

1. Arias CA, Murray BE. Antibiotic-resistant bugs in the 21 st century: A clinical super challenge. $\mathrm{N}$ Eng J Med 2009:360(5):439-443. [http://dx.doi.org/10.1056/NEJMp0804651]

Zapun A, Contreras-Martel C, Vernet T. Penicillin-binding proteins and beta-lactam resistance. FEMS Microbiol Rev 2008;32(2):361-385. [http://dx.doi.org/10.1111/j.1574-6976.2007.00095.x]

Bush K. Alarming $\beta$-lactamase-mediated resistance in multidrug-resistant Enterobacteriaceae. Curr Opin Microbiol 2010;13(5):558-564. [http://dx.doi.org/10.1016/j.mib.2010.09.006]

4. Ahmed AA, Osman H, Mansour AM, et al. Antimicrobial agent resistance in bacterial isolates from patients with diarrhea and urinary tract infection in the Sudan. Am J Trop Med Hyg 2000;63(5-6):259263.

Mandomando I, Sigaúque B, Morais L, et al. Antimicrobial drug resistance trends of bacteremia isolates in a rural hospital in southern Mozambique. Am J Trop Med Hyg 2010; 83(1):152-157. [http:// dx.doi.org/10.4269/ajtmh.2010.09-0578

6. Apalata T, Zimba TF, Sturm WA, Moodley P. Antimicrobial susceptibility profile of Neisseria gonorrhoeae isolated from patients attending a STD facility in Maputo, Mozambique. Sex Transm Dis 2009;36(6):341-343. [http://dx.doi.org/10.1097/OLQ.0b013e3181982e3c] 
7. Mandomando IM, Macete EV, Ruiz J, et al. Etiology of diarrhea in children younger than 5 years of age admitted in a rural hospital of southern Mozambique. Am J Trop Med Hyg 2007;76(3):522-527. 8. Tessema B, Kassu A, Mulu A, Yismaw G. Predominant isolates of urinary tract pathogens and thei 8. Tessema B, Kassu A, Mulu A, Yismaw G. Predominant isolates of urinary tract pathogens and thei antimicrobial susceptibility in Gondar University Teaching Hospital, northwest Ethiopia. Ethiop Med 2007;45(1):61-67.

9. Aboderin OA, Abdu AR, Odetoyin BW, Lamikanra A. Antimicrobial resistance in Escherichia coli strains from urinary tract infections. J Natl Med Assoc 2009;101(12):1268-1273

10. Bercion R, Mossoro-Kpinde D, Manirakiza A, Le Faou A. Increasing prevalence of antimicrobial resistance among Enterobacteriaceae uropathogens in Bangui, Central African Republic. J Infect Dev Ctries 2009;3(3):187-190.

11. Habte TM, Dube S, Ismail N, Hoosen AA. Hospital and community isolates of uropathogens at a tertiary hospital in South Africa. S Afr Med J 2009;99(8):584-587.

2. Pezzlo M, York MK. Clinical Microbiology Procedures Handbook. 2nd ed. Washington DC: ASM Press, 2004:3.12.1-3.12.31

13. al Naiemi N, Cohen SJ, Leverstein-van Hall M. NVMM-richtlijn voor screening en confirmatie van extended-spectrum bètalactamases in Enterobacteriaceae. Ned Tijdschr Med Microbiol 2008;16(2):23-28.

14. Workingparty Infection Prevention. Measures to prevent transmission of highly resistant microorganisms (HRMO). http://www.wip.nl/UK/free_content/Richtlijnen/HRMO.pdf (accessed 10 microorganisms

15. Manthous CA, Amoateng-Adjepong Y. Empiric antibiotic use and resistant microbes: a 'Catch-22' for the 21 st century. Chest 2000;118(1):9-11. [http://dx.doi.org/10.1378/chest.118.1.9.]
16. Kala UK, Jacobs DWC. Evaluation of urinary tract infection in malnourished black children. Ann Trop Paediatr 1992;12(1):75-81.

17. Gad GF, Mohamed HA, Ashour HM. Aminoglycoside resistance rates, phenotypes, and mechanisms of Gram-negative bacteria from infected patients in upper Egypt. PLoS One 2011;6(2):e17224. [http:// dx.doi.org/10.1371/journal.pone.0017224]

18. Kotra LP, Haddad J, Mobashery S. Aminoglycosides: perspectives on mechanisms of action and resistance and strategies to counter resistance. Agents Chemother 2000;44(12):3249-3256. [http:// dx.doi.org/10.1128/AAC.44.12.3249-3256.2000]

19. Barbosa TM, Levy SB. The impact of antibiotic use on resistance development and persistence. Drug Resist Updat 2000;3(5):303-311. [http://dx.doi.org/10.1054/drup.2000.0167]

20. World Health Organization. Management of Severe Malnutrition: a Manual for Physicians and other Senior Health Workers. http://whqlibdoc.who.int/hq/1999/a57361.pdf (accessed 10 January 2012).

21. Asharam K, Bhimma R, Adhikari M. Human immunodeficiency virus and urinary tract infections in children. Ann Trop Paediatr 2003;23(4):273-277. [http://dex.doi.org/10.1179/027249303225007653]

22. Leverstein-van Hall, Dierikx CM, Cohen Stuart J, et al. Dutch patients, retail chicken meat and poultry share the same ESBL genes, plasmids and strains. Clin Microbiol Infect 2011;17(6):873-880. [http:// dx.doi.org/10.1111/j.1469-0691.2011.03497.x]

23. Falagas ME, Kopterides P. Risk factors for the isolation of multi-drug-resistant Acinetobacter baumannii . Falagas ME, Kopterides P. Risk factors for the isolation of multi-drug-resistant Acinetobacter baumannii
and Pseudomonas aeruginosa: a systematic review of the literature. J Hosp Infect 2006;64(1):7-15. [http:dx.doi.org/10.1016/j.jhin.2006.04.015] 